\title{
Analysis of MEA's durability under Accelerated Stress Tests that mimic realistic automotive operations
}

\author{
Elena Colombo*, Andrea Baricci, and Andrea Casalegno \\ Politecnico di Milano, Department of Energy, Via Lambruschini 4, 20156 Milano, Italy
}

\begin{abstract}
This work aims at studying MEA's ageing under single operational "mode" accelerated stress tests (AST), that were specifically designed to replicate under hydrogen/air feeding the main stressors of realistic operations in the automotive sector. A methodology for developing AST is here presented and preliminary results about the activity included. In particular, low power and high power functioning have been mimicked in a Zero-Gradient hardware, which allows a reliable materials comparison. Quantities, measurable in-situ and operando, have been tracked during ageing, like cell power, polarization curves, Pt active area, oxygen mass transport resistance, Electrochemical Impedance Spectra. The final objective is to clarify the underlying ageing mechanisms and assess the contribution of each specific operation to the MEA lifetime, focusing in particular on the cathode catalyst layer durability. Moreover, the rate of voltage loss for the new ASTs has been successfully correlated to the degradation observed under a complete driving cycle protocol.

Index Terms - Accelerated Stress Test, Automotive, Degradation mechanisms, PEMFC, Catalyst durability.
\end{abstract}

\section{Introduction}

The typical conditions of automotive driving cause durability issues that it is necessary to overcome for a large-scale commercialization of Polymer Electrolyte Membrane Fuel Cells (PEMFC). The operating state affects lifetime since it degrades the different components of PEMFC: many degradation mechanisms could occur ${ }^{12}$, which have been extensively studied and recognized. However, it is not clear how much they contribute to real ageing, considering in particular the mitigation strategies that are applied today in vehicles: identify such contributions in dependence on the operational modes is crucial for orienting the direction of research and development activities. This work presents a methodology for developing Accelerated Stress Tests (AST) for single cell (SC) starting from a realistic driving cycle. The reference driving cycle, used for designing the operative conditions of ASTs and to guarantee their validation, was obtained starting from vehicles data in the frame of the EU Horizon 2020 ID-FAST project (grant agreement No 779565) ${ }^{3}$. The observed and measured clue stressors of realistic operations have been successfully replicated on a Zero-Gradient hardware, by separating specific "operational modes". In particular, different state-of-the-art Membrane Electrode Assemblies (MEA's) were tested under ASTs that mimic low and high power automotive functioning, as well as under a suitable combination of these protocols, with the purpose of resembling realistic ageing and identifying correlations in terms of degradation rates of clue quantities (like $i-V$ curves performance and $\mathrm{Pt}$ active area loss).

\section{Experimental}

\subsection{Tools and MEA characterization}

A Zero-Gradient hardware ${ }^{4,5,6}$, developed at Politecnico di Milano, was adopted for testing different materials in a reliable way, ensuring homogenous conditions over the cell active area (from 2 to $10 \mathrm{~cm}^{2}$ ). Its purpose is to minimize the influence from the testing hardware (e.g. flowfiled design) on results of performance and durability protocols. A high and accurate control of the operating conditions is guaranteed. To investigate the alterations induced by the studied protocols, electrochemical characterization methods were exploited: performance polarization curves (both under $\mathrm{H}_{2} /$ air and $\mathrm{H}_{2} / \mathrm{O}_{2}$ ), electrochemical impedance spectroscopy (under $\mathrm{H}_{2} /$ air, $\mathrm{H}_{2} / \mathrm{O}_{2}$ and $\mathrm{H}_{2} / \mathrm{N}_{2}$ ), cyclic voltammetry, linear sweep voltammetry and limiting current measurement, following a protocol close to Baker et al. ${ }^{7}$. Reference polarization curve operative conditions in $\mathrm{H}_{2} /$ air are: 80 ${ }^{\circ} \mathrm{C}$, fully humidified gases both at anode and cathode, $\mathrm{P}_{\mathrm{c}} / \mathrm{P}_{\mathrm{a}}=230 / 250 \mathrm{kPa}$. Stoichiometry is set equal to 8 and $>10$ at anode and cathode respectively in case of ZeroGradient hardware. Limiting current measurement, under excess gas feeding, was obtained at different pressures

\footnotetext{
* Corresponding author: elena.colombo@polimi.it
} 
$\left(\mathrm{P}_{\mathrm{c}} / \mathrm{P}_{\mathrm{a}}=150 / 170 \mathrm{kPa}, 250 / 270 \mathrm{kPa}, 350 / 370 \mathrm{kPa}\right)$ and diluted percentages of dry oxygen in nitrogen $(1 \%, 2 \%$ and $3 \%)$. The results of the ASTs activity are compared to those of MEA's aged under conditions closer to stack operation. In particular, the realistic ID-FAST driving cycle protocol, used as a starting point for the ASTs design and for their validation, was carried out on a 25 $\mathrm{cm}^{2}$ macro-segmented hardware. Hardware details are provided in references ${ }^{8,9,4,10}$.

\subsection{Tested MEA's and protocols}

Different Pt/C MEA's were tested: state-of-of-the-art materials with different catalytic loading, ionomer and support type have been compared. They provide more generality to the drawn conclusions and highlighted possible differences during ageing among MEA's which were similarly performant at BoL (Beginning of Life). MEA's were degraded under newly developed Accelerated Stress Tests. A new ad-hoc protocol, designed for the specific process of air start-up, was developed and presented in a previous work $^{4}$. It introduced realistic stressors (i.e. high potentials, up to $1.5 \mathrm{~V}$, but ambient temperature and very fast transients) according to the mitigation strategies commonly adopted in nowadays vehicles. Bisello et al. successfully correlated the protocol to the realistic process. Here, another AST, named Low Power AST (LP AST), was designed to specifically mimic the low power operation. It was carried out in galvanostatic mode under hydrogen/air. For each MEA, the current densities correspondent to $0.85 \mathrm{~V}$ and $0.70 \mathrm{~V}$ have been evaluated at the BoL: voltages were selected in order to be close to real systems. Then, these current densities were imposed as a stressor for studying degradation. A single low power cycle is formed by: a basic unit of $30 \mathrm{~s}$ at the minimum current (i.e. $0.85 \mathrm{~V}$ at BoL) and $12 \mathrm{~s}$ at the high current (i.e. $0.7 \mathrm{~V}$ at $\mathrm{BoL}$ ); the unit is repeated 6 times and it is finally followed by $30 \mathrm{~s}$ at the minimum current. After each set of current cycling, a short stop (110 s) is introduced. It causes voltage drop to very low values $(<$ $0.1 \mathrm{~V})$, that reduces the Pt surface, as well as pressure cycles due to the interruption of the cathodic flux. Cell temperature was set equal to $71{ }^{\circ} \mathrm{C}, \mathrm{RH}_{\mathrm{A}} / \mathrm{RH}_{\mathrm{C}}=60 / 70 \%$ and $\mathrm{P}_{\mathrm{A}, \text { in }} / \mathrm{P}_{\mathrm{C} \text {,in }}=190 / 140 \mathrm{kPa}$. Conditions were selected such to replicate the "mean" state of a MEA subjected to driving cycle. Constant fluxes were set such to ensure $\lambda_{A} / \lambda_{C}=8 / 20$ at the maximum current. High Power AST (HP AST) introduces the main stressors of the high load operation. Again, the AST aims at reproducing the voltage cycling observed in a realistic process but exploiting galvanostatic mode in order to make the operation closer to actual systems and check the voltage decay in time. The current densities were defined such to correspond to $0.85 \mathrm{~V}, 0.75 \mathrm{~V}, 0.7 \mathrm{~V}, 0.65 \mathrm{~V}$ at BoT. Pressure cycles up to $\mathrm{P}_{\mathrm{A}, \text { in }} / \mathrm{P}_{\mathrm{C} \text {,in }}=300 / 280 \mathrm{kPa}$ and gas switch from humidified $\left(\mathrm{RH}_{\mathrm{A}} / \mathrm{RH}_{\mathrm{C}}=60 / 70 \%\right)$ to completely dry were included. Cell temperature was set equal to $90^{\circ} \mathrm{C}$. Every 200 cycles, a long stop have been simulated by cooling down the system for $12 \mathrm{~h}$. Then, electrochemical and performance characterization were carried out. Preliminary results about these ASTs have been obtained for a total of 1000 cycles (approximately $120 \mathrm{hrs}$ of testing for Low Power AST and $70 \mathrm{hrs}$ for High Power AST). A Combined AST, which alternates Low and High Power, was tested too, in order to take care of the interplay between the two types of operation. Finally, the Combined AST was validated: correlations to the decay of performance and of the main electrochemical parameters of real world functioning was identified such to quantify the acceleration factors. It should be also proved that the new design does not alter the ageing mechanisms involved in realistic processes. Preliminary results about this point have been obtained but future activity will furnish further insights.

\section{Results and discussion}

The design of single "mode" accelerated stress tests was carried out starting from the driving cycle protocol defined in ID-FAST project. The Low Power AST was intended to include voltage cycling as the main stressors, which is consequence of load changes and short stops: a $\mathrm{x} 7$ acceleration factor was introduced by reducing the holding times compared to the realistic profile. The operando High Frequency Resistance (HFR approximated with impedance measured at $1000 \mathrm{~Hz}$ ) was proved to be comparable to the value observed in the middle region of the MEA operated under the same kind of operation in the driving cycle. Pressure is set at the level of $140 / 190 \mathrm{kPa}$ during the load cycling, while it decreases every short stop. Voltage cycling due to load changes is recognized to play a key role in cathode catalyst layer ageing since it causes Pt active area loss through mechanisms like Pt dissolution 11,12,13; therefore, a sufficiently accurate reproduction of this stressor was considered as fundamental. The High Power AST was designed with the purpose of mimicking both voltage cycling and MEA hydration cycles, that were verified as relevant in the transitions from low to high loads. Pressure cycles were introduced as well. Pressure makes wide cycles at cathode between ambient value (short stop) up to $280 \mathrm{kPa}$, while between $150 \mathrm{kPa}$ (short stop) to $300 \mathrm{kPa}$. Again, a $\mathrm{x} 7$ time acceleration factor was introduced. As done in case of Low Power, the MEA hydration state was verified as coherent to middle region of the realistic driving cycle. At the first stage of each High Power cycle, thanks to the growing pressure and the increasing current setpoints, the HFR drops significantly. Thereafter, the introduced drier gas feeding, combined with a decreasing load setpoint and decreasing pressure, the HFR very steeply grows. The process is repeated every High Power cycle, introducing thus hydration cycles that are expected to cause a mechanical stress for membrane and/or ionomer in the catalyst layer ${ }^{14,15}$. Finally, the Combined AST was realized by alternating Low and High Power AST. Preliminary results revealed that the two processes could influence one each other, therefore an alternation of 200 cycles +200 cycles was kept. All the AST protocols cause an analogous decay of the Pt active area: largest losses occurred during the first 200 cycles and the Pt active area (ECSA) loss stabilizes 
in the range of $30 \% \div 40 \%$ for all the tested materials. Checking the operando voltage profiles, the largest part of the loss is recovered by the way of long stops ${ }^{16}$. As preliminary observation, $i-V$ performance, recorded during characterization, shows that discrepancies among the materials are minor under Low Power AST: all of them present a downwards vertical translation as the most relevant ageing impact and changes in mass transport resistance could be correlated to ECSA loss ${ }^{17}$. High Power AST was verified to play a major role in ageing rather than low power operation, even though the MEA operates in this condition for less time (operative time of 1 High Power AST cycle $\approx 60 \% 1$ Low Power AST cycle). Clear differences in terms of performance loss rise among the materials under this kind of operation. In addition, the voltage profile keeps a higher rate of loss at high current densities. Some evidences indicate an increased ohmic loss.

Combining the protocols, there is no linear summation of the performance loss rates. Indeed, ECSA reaches a stabilization, that makes the voltage losses at low current values comparable among all the developed protocols. Performance decay at high current densities is instead controlled by the high power operation. As a validation of the developed design, it was proved that 1 cycle of the Combined AST matches the average losses caused by 1 operative hour of the realistic protocol: reasonable consistency was verified in terms of ECSA decay, performance loss and oxygen mass transport resistance evolution. In particular, adopting the reference polarization curve and the reference material, the rate of voltage loss was $10.8 \mu \mathrm{V} /$ cycle in the AST case versus $8.0 \mu \mathrm{V} / \mathrm{hr}$ of the global driving cycle protocol loss, at the current setpoint that corresponds to $0.78 \mathrm{~V}$ at BoL. At larger current densities (setpoint equivalent to $0.65 \mathrm{~V}$ at $\mathrm{BoL}$ ) the AST rate of $30.5 \mu \mathrm{V} /$ cycle matches the realistic $28.8 \mu \mathrm{V} / \mathrm{hr}$. Voltage loss comparison between the two testing activities is collected in Fig.1, while compatibility of catalyst active area drop is shown in Fig.2.
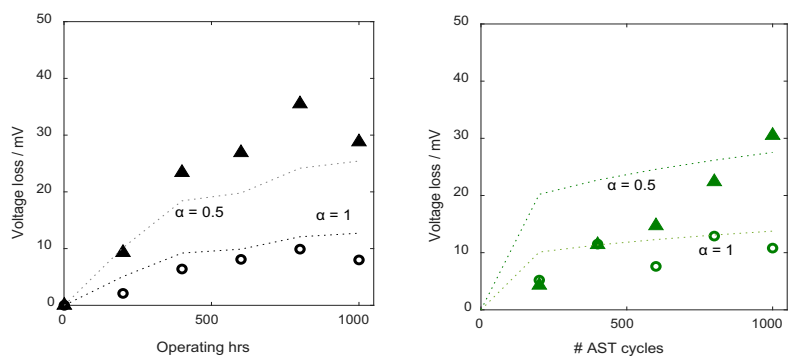

Fig. 1 (left) Voltage loss computed as the difference between the performance at BoT and after 1000 operating hours under ID-FAST driving cycle protocol ${ }^{3}$, measured under $\mathrm{H}_{2} /$ air, $\mathrm{T}=$ $80{ }^{\circ} \mathrm{C}$, fully humidified conditions, $\mathrm{P}_{\mathrm{A}, \text { in }} / \mathrm{P}_{\mathrm{C}, \text { in }}=230 / 250 \mathrm{kPa}, \lambda_{\mathrm{A}} /$ $\lambda_{\mathrm{C}}=2 / 4$. Adopted hardware: triple-serpentine $25 \mathrm{~cm}^{-2}$ single cell. Symbols: triangle indicates current density 2 and circle current density 1 (see Table 1); (right) Voltage loss computed as the difference between the performance at BoT and after 1000 AST cycles, measured under $\mathrm{H}_{2} /$ air, $\mathrm{T}=80^{\circ} \mathrm{C}$, fully humidified conditions, $\mathrm{P}_{\mathrm{A}, \mathrm{in}} / \mathrm{P}_{\mathrm{C}, \text { in }}=230 / 250 \mathrm{kPa}, \lambda_{\mathrm{A}} / \lambda_{\mathrm{C}}=8 / 20$. Adopted hardware: Zero-Gradient cell. Symbols: triangle indicates current density 2 and circle current density 1 (see Table 1). Results are reported for one of the tested MEA's.

\begin{tabular}{|l|l|c|c|}
\hline & i / $\mathbf{A ~ \mathbf { ~ m } ^ { - 2 }}$ & $\begin{array}{c}\text { Voltage at } \\
\text { BoT }\end{array}$ \\
\hline Zero-G & $\begin{array}{l}\text { Current } \\
\text { density 1 }\end{array}$ & 0.7 & $0.78 \mathrm{~V}$ \\
\cline { 2 - 4 } & $\begin{array}{l}\text { Current } \\
\text { density 2 }\end{array}$ & 2.8 & $0.65 \mathrm{~V}$ \\
\hline $\mathbf{2 5} \mathbf{c m}^{\mathbf{2}}$ cell & $\begin{array}{l}\text { Current } \\
\text { density 1 }\end{array}$ & 0.7 & $0.78 \mathrm{~V}$ \\
\cline { 2 - 4 } & $\begin{array}{l}\text { Current } \\
\text { density 2 }\end{array}$ & 1.9 & $0.65 \mathrm{~V}$ \\
\hline
\end{tabular}

Table 1. Current densities values used for comparison of Combined AST and driving cycle results, as indicated in Fig. 1.

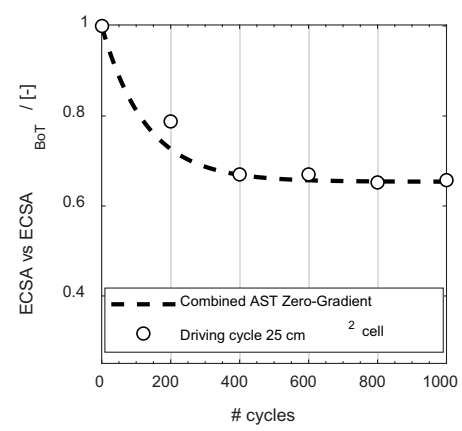

Fig. 2 Evolution of Pt active area (ECSA) with the number of AST cycles/operating hours for Combined AST and driving cycle protocol respectively. ECSA is measured from cyclic voltammetry: $\mathrm{H}_{2} / \mathrm{N}_{2}$ flow $=60 / 60 \mathrm{ml} \mathrm{min}{ }^{-1}, \mathrm{RH} 100 / 100$, scan rate $0.05 \mathrm{~V} \mathrm{~s}^{-1}, 30^{\circ} \mathrm{C}$, ambient pressure, minimum potential $=$ $0.079 \mathrm{~V}$ and maximum potential of $0.6 \mathrm{~V}$. Results are reported for one of the tested MEA's.
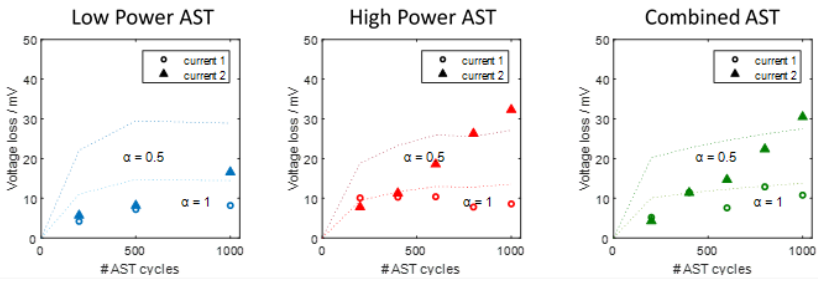

Fig. 3 Voltage loss computed as the difference between the performance at BoT and after 1000 AST cycles. The three developed ASTs are compared: Low Power (left), High Power (middle) and Combined (right). Performance loss is measured under $\mathrm{H}_{2} /$ air, $\mathrm{T}=80{ }^{\circ} \mathrm{C}$, fully humidified conditions, $\mathrm{P}_{\mathrm{A}, \text { in }} / \mathrm{P}_{\mathrm{C}, \text { in }}=$ $230 / 250 \mathrm{kPa}, \lambda_{\mathrm{A}} / \lambda_{\mathrm{C}}=2 / 4$. Adopted hardware: Zero-Gradient cell. Symbols: triangle indicates current density 2 and circle current density 1 (see Table 1). Results are reported for one of the tested MEA's.

\section{Conclusions}

A methodology for designing new Accelerated Stress Tests protocols that ensure real operations representativeness has been presented. A process of 
identification of the main stressors has been carried out. Each operational "mode" has been studied in a separate way and finally combined to resemble the complete driving cycle ageing. The final objective is to identify an acceleration rate without altering the realistic degradation mechanisms. New ASTs representatives has been preliminary proved and they revealed as an effective tool for clarifying the underlying degradation mechanisms under each process. In particular, as evinced in Fig.3, at Low Power conditions, the voltage loss in time reaches almost a stabilization both at low and high current densities of $i$ - $V$ curve, with largest drops in the first 200 AST cycles. The result is consistent to the profile of ECSA decay and to the consequent activation overpotential (Fig.2). Under High Power AST and Combined AST, the rate of voltage loss at high currents keeps greater, indicating an additional mechanism of degradation under high load operation, probably caused by mechanical stress. Finally, it was proved that 1 cycle of the Combined AST represents well 1 cycle of the realistic driving cycle protocol (Fig. 1 and Fig. 2). Future work will consist in analysing a larger number of cycles for the newly developed AST protocols and in further comprehending the mechanisms responsible of ageing through materials comparison and ex-situ analysis, that will help in validating the transfer function to "real world" too.

\section{Acknowledgment}

The work received funding under ID-FAST grant agreement No 779565. This Joint Undertaking receives support from the EU Horizon 2020 program

\section{Bibliography}

1. P. Ren, P. Pei, Y. Li, Z. Wu, D. Chen, and S. Huang, Prog. Energy Combust. Sci., 80, 100859 (2020).

2. T. Zhang, P. Wang, H. Chen, and P. Pei, Appl. Energy, 223, 249-262 (2018).

3. F. Wilhelm, S. Escribano, et al, ID-FAST Investigations on degradation mechanisms and Definition of protocols for PEM Fuel cells Accelerated Stress Testing; Grant agreement no : 779565; Call : H2020-JTIFCH-2017-1; D4.3 - Analysis of coupling between mechanisms and definition of comb., (2021).
4. A. Bisello, E. Colombo, A. Baricci, C. Rabissi, L. Guetaz, P. Gazdzicki, and A. Casalegno, J. Electrochem. Soc., 168, 054501 (2021).

5. T. Bednarek and G. Tsotridis, Data in Bief, 31, 105945 (2020).

6. T. Bednarek and G. Tsotridis, J. Power Sources, 473, 228319 (2020).

7. D. R. Baker, D. A. Caulk, K. C. Neyerlin, and M. W. Murphy, J. Electrochem. Soc., 156, B991 (2009).

8. C. Rabissi, P. Gazdzicki, L. Guétaz, S. Escribano, L. Grahl-Madsen, A. Baricci, and A. Casalegno, J. Power Sources, 397, 361-373 (2018).

9. C. Rabissi, E. Brightman, G. Hinds, and A. Casalegno, Int. J. Hydrogen Energy, 43, 9797-9802 (2018).

10. E. Colombo, A. Bisello, A. Casalegno, and A. Baricci, J. Electrochem. Soc., 168, 054508 (2021).

11. P. Zihrul, I. Hartung, S. Kirsch, G. Huebner, and H. A. Gasteiger, 163, 492-498 (2016).

12. W. Bi and T. F. Fuller, 178, 188-196 (2008).

13. P. P. Lopes, D. Tripkovic, P. F. B. D. Martins, D. Strmcnik, E. A. Ticianelli, V. R. Stamenkovic, and N. M. Markovic, J. Electroanal. Chem., 819, 123-129 (2018).

14. A. Kusoglu, A. M. Karlsson, M. H. Santare, S. Cleghorn, and W. B. Johnson, J. Power Sources, 161, 987-996 (2006).

15. T. Morawietz, M. Handl, C. Oldani, P. Gazdzicki, J. Hunger, F. Wilhelm, J. Blake, K. A. Friedrich, and R. Hiesgen, J. Electrochem. Soc., 165, F3139-F3147 (2018).

16. P. Gazdzick, J. Mitzel, D. Sanchez, M. Schulze, and K. A. Friedrich, J. Power Sources, 327, 86-95 (2016).

17. T. A. Greszler, D. Caulk, and P. Sinha, J. Electrochem. Soc., 159, F831-F840 (2012). 